\title{
Free Volume Power Law for Transport Properties of Hard Sphere Fluid
}

\author{
Hongqin $\operatorname{Liu}^{1}$ \\ ${ }^{1}$ Shared Services Canada
}

November 9, 2020

\begin{abstract}
This paper presents a study on the relationship between transport properties and geometric free volume for dense hard sphere (HS) systems. Firstly, a generic free volume distribution function is proposed based on recent molecular dynamic (MD) simulations for the HS geometric free volume1,2. Combining the new distribution function with a local particle transportation model, we obtain a power law for the HS transport properties. Then a relation between the geometric free volume and thermodynamic free volume is established, which makes it possible to obtain the expressions of the geometric free volume. The new models are tested with MD results for HS viscosity, diffusivity, respectively and the results are very satisfactory. Using the power law we are able to reproduce equations obtained from different approaches, such as the entropy scaling laws3, mode coupling theory4 or empirical correlations5. In particular, A long-standing controversy regarding the Cohen-Turnbull-Doolittle free volume model6,7 is resolved.
\end{abstract}

\section{Hosted file}

HS transport properties power law AIChEJ.pdf available at https://authorea.com/users/371423/ articles/491620-free-volume-power-law-for-transport-properties-of-hard-sphere-fluid 


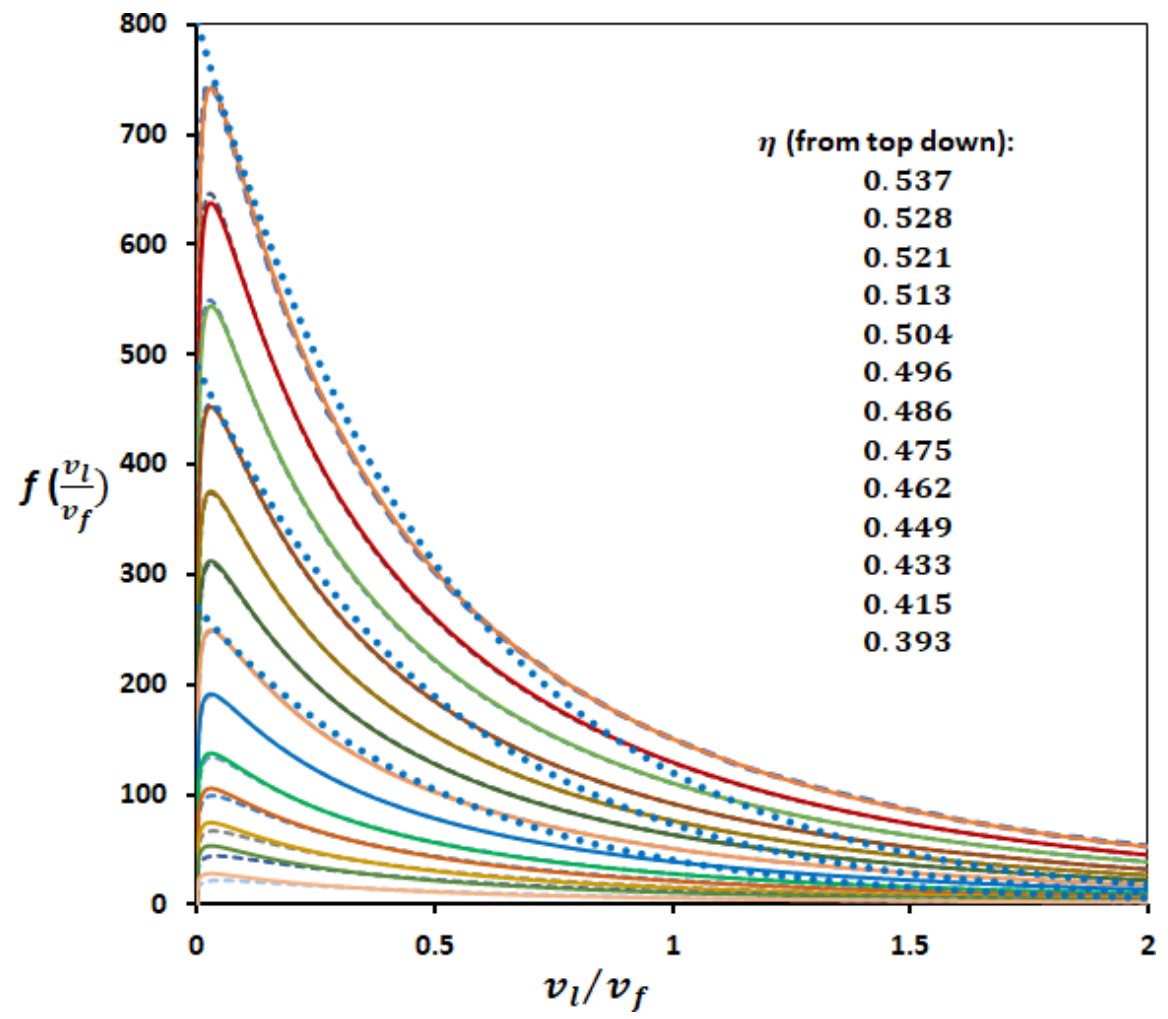

$$
\ln \left(\frac{v_{t f}}{v}\right)
$$

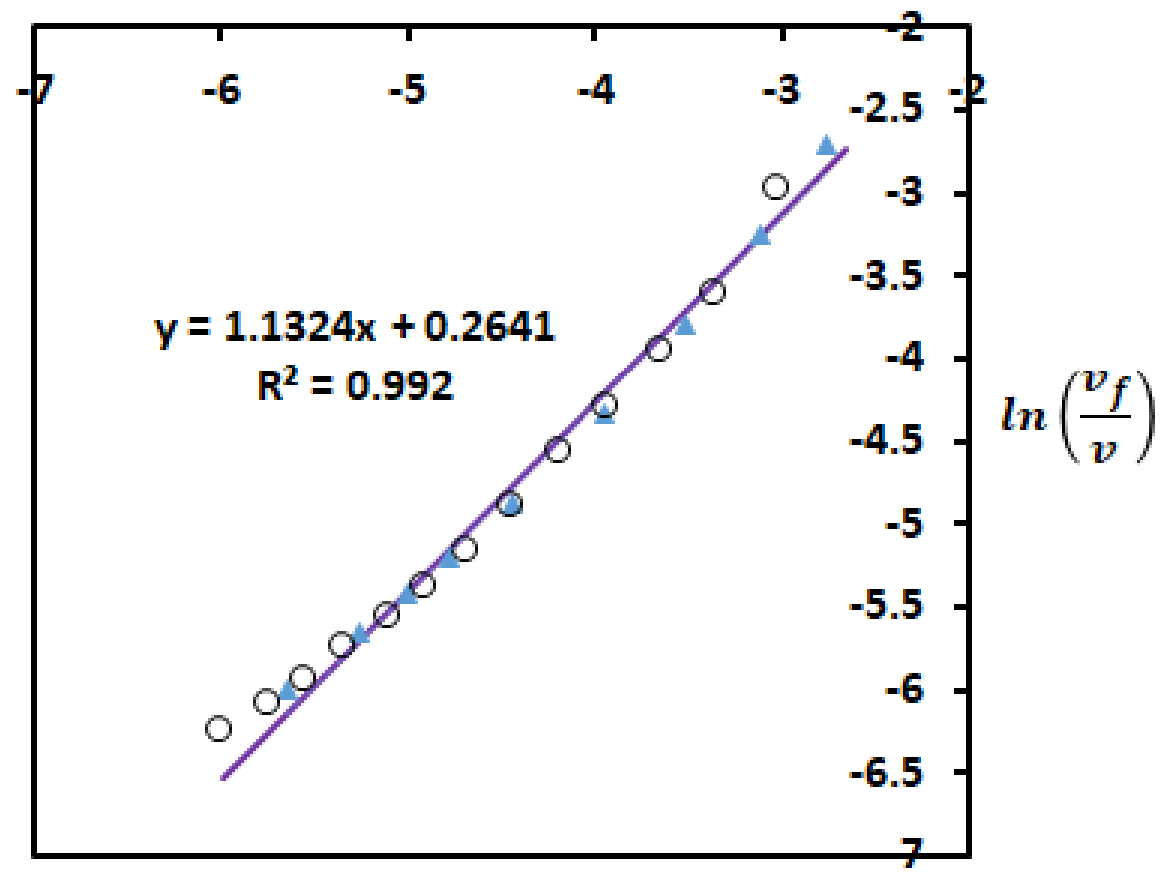



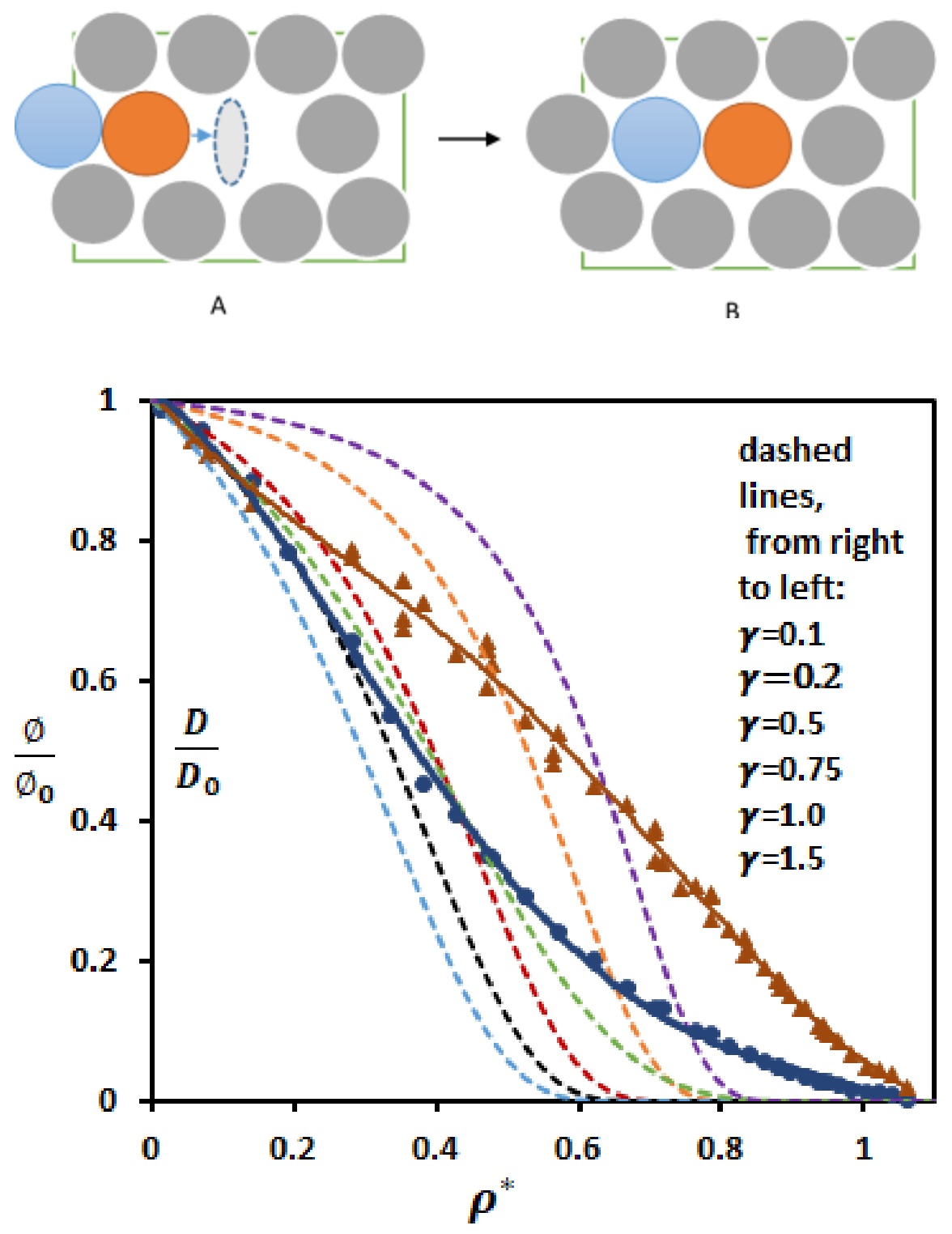

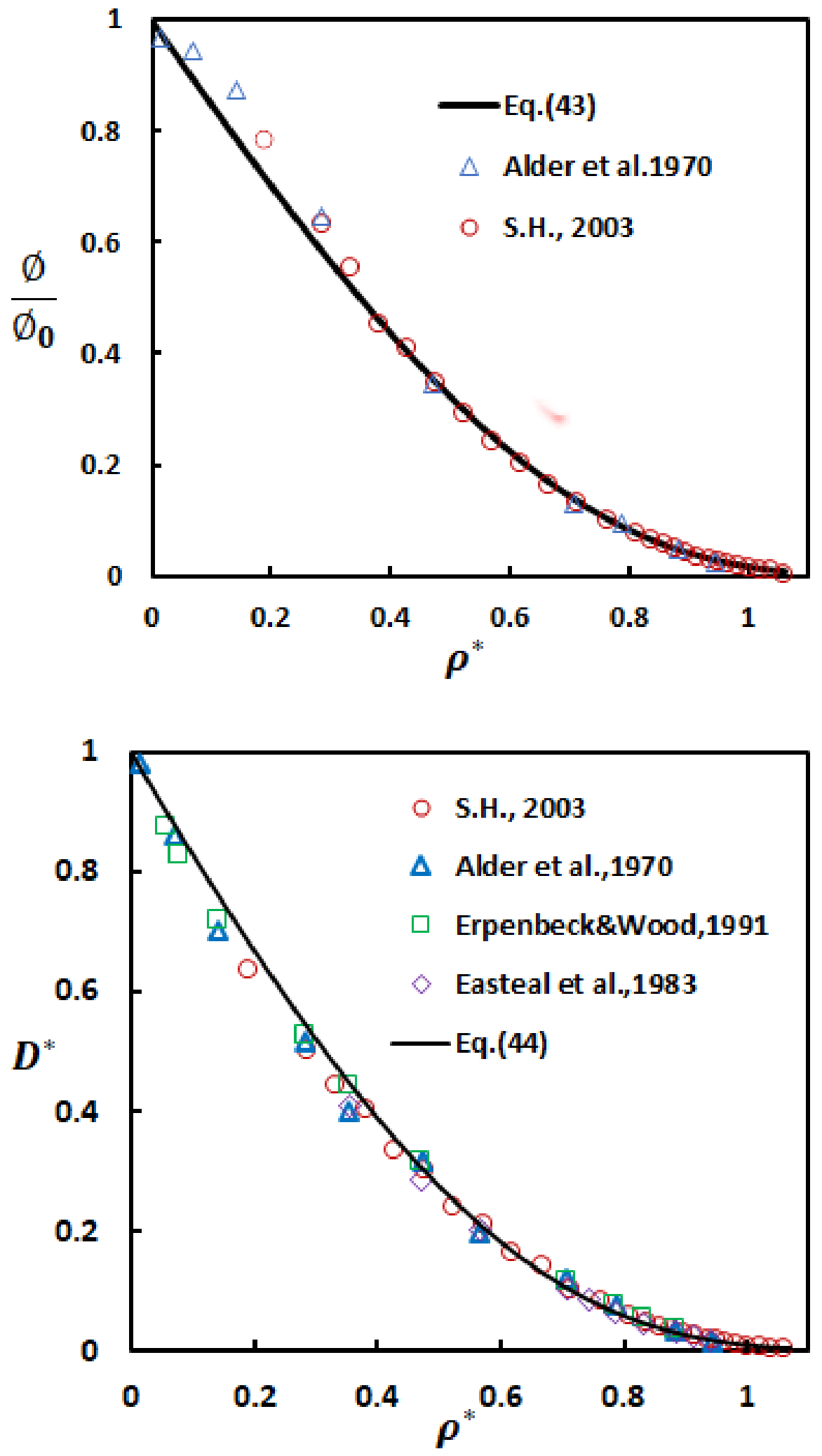

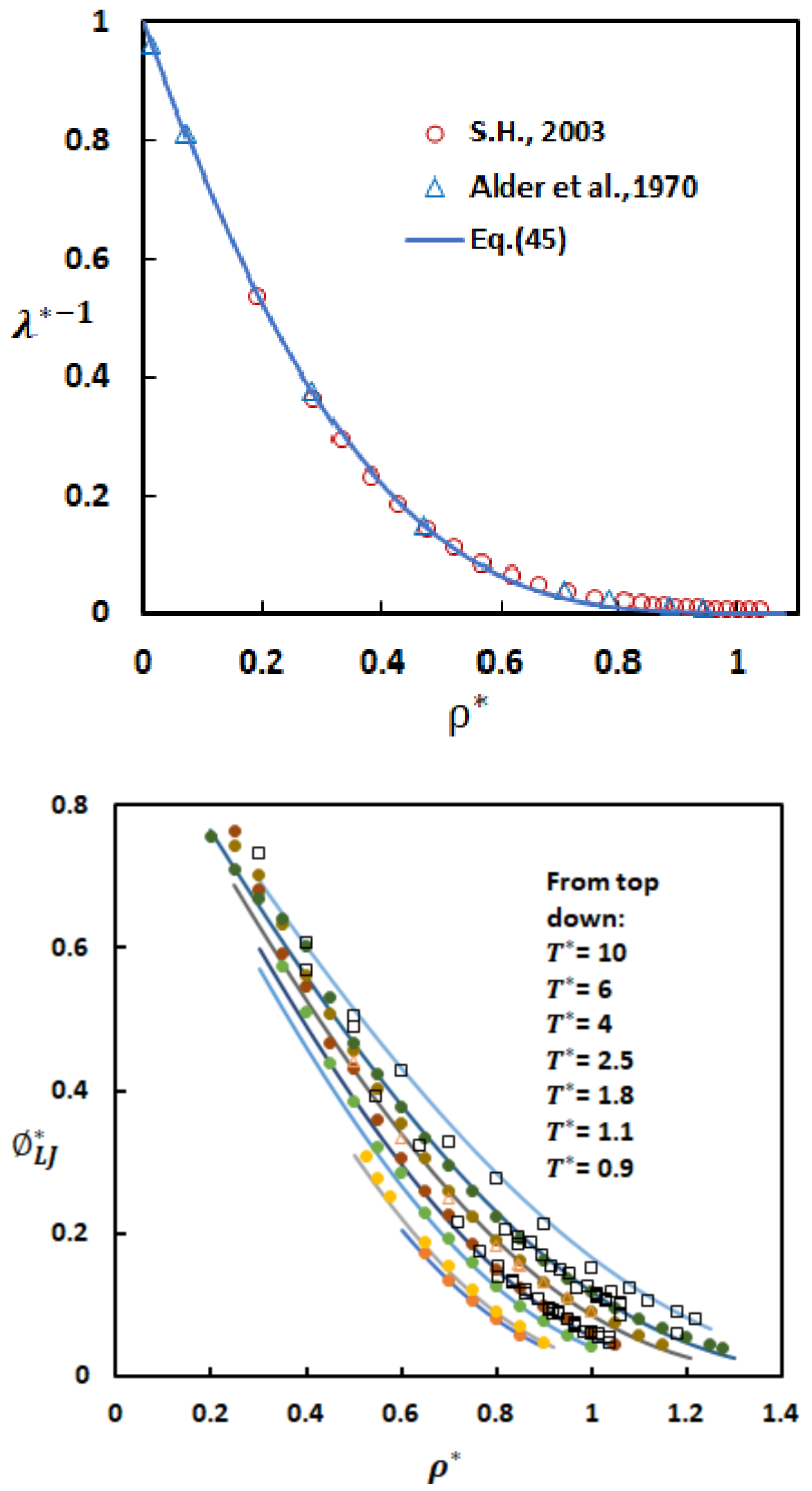

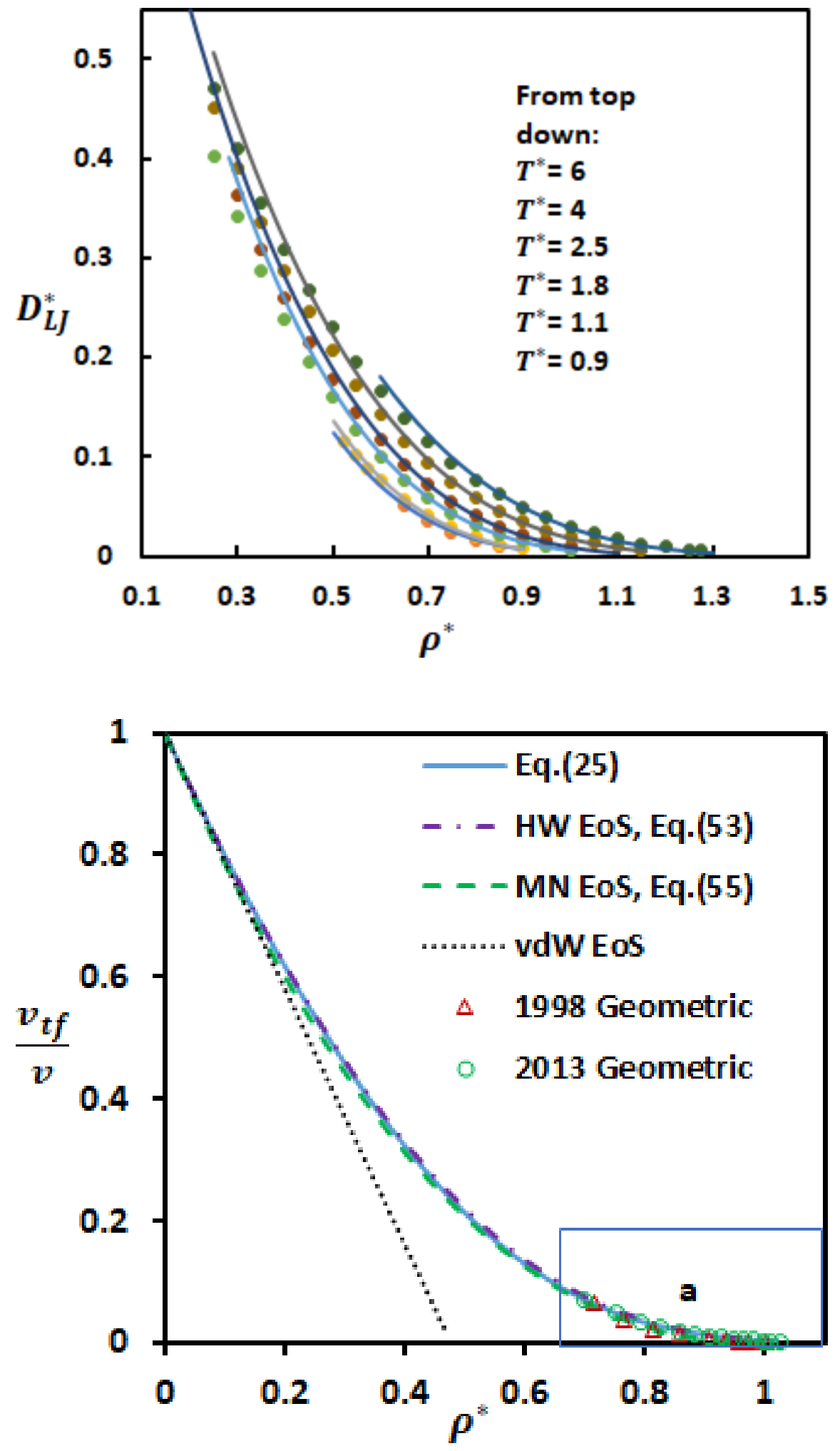


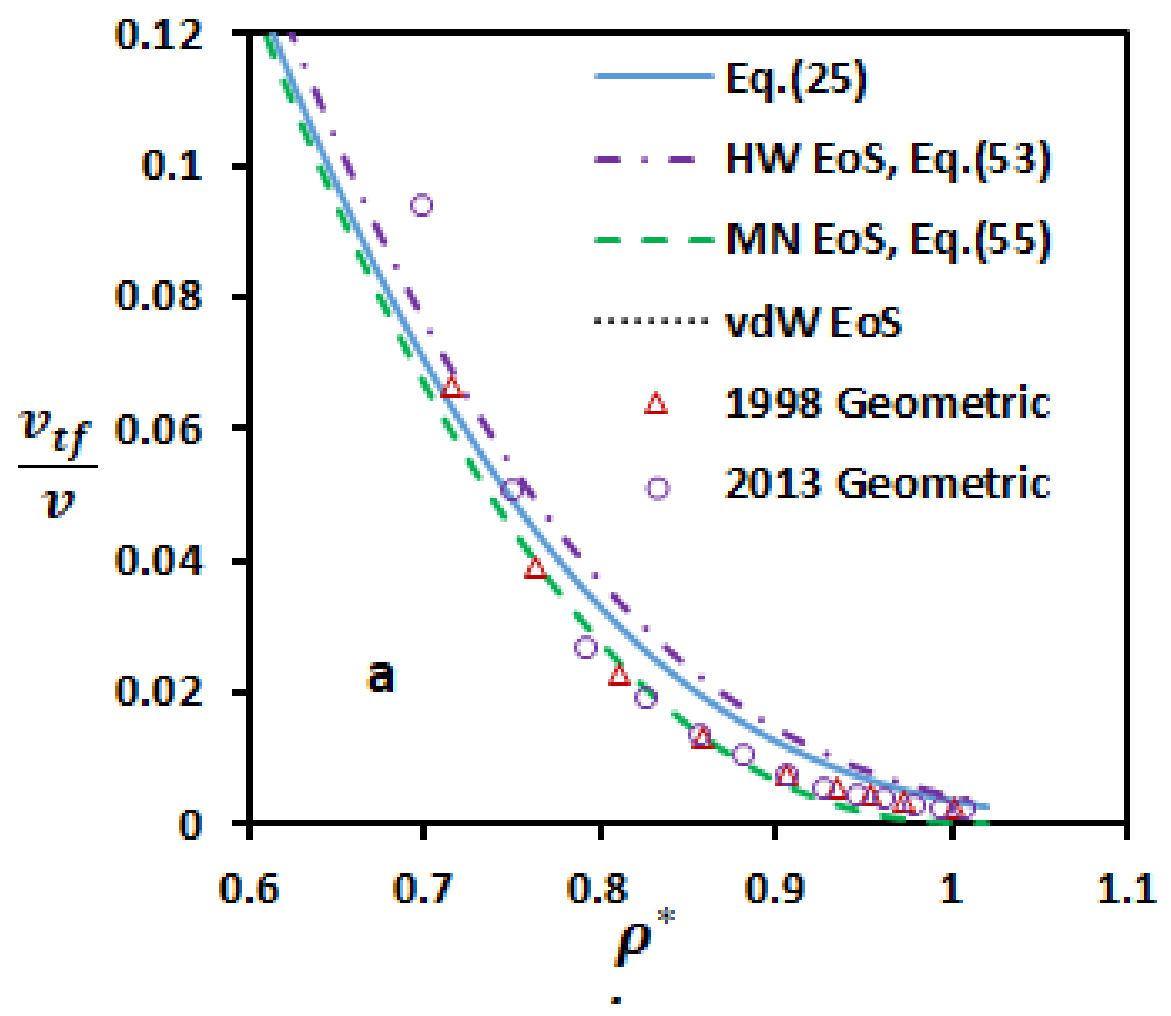

